Original article

\title{
Classical swine fever in Victorian domestic pigs: Evidence of disease freedom
}

\author{
JC Hunnam $^{\mathrm{a}^{*}}$, K Moore ${ }^{\mathrm{a}}$, P Daniel ${ }^{\mathrm{a}}$, MA Stevenson ${ }^{\mathrm{b}}$, SE Salmon ${ }^{\mathrm{a}}$
}

a Chief Veterinary Officer's Unit, Agriculture Victoria, Department of Economic Development, Jobs, Transport and Resources, 475 Mickleham Road, Attwood Victoria 3049, Australia

${ }^{\mathrm{b}}$ Faculty of Veterinary and Agricultural Sciences, The University of Melbourne, Parkville Victoria 3010 Australia

*Corresponding author: jaimie.hunnam@ecodev.vic.gov.au

This is the author manuscript accepted for publication and has undergone full peer review but has not been through the copyediting, typesetting, pagination and proofreading process, which may lead to differences between this version and the Version of Record. Please cite this article as doi: 10.1111 /avj.12871

This article is protected by copyright. All rights reserved. 


\begin{abstract}
Australia is currently regarded as free of classical swine fever (CSF), a highly contagious disease of pigs caused by a pestivirus. To provide additional evidence that the Victorian domestic pig population is free of CSF, 391 pigs from 23 holdings were sampled at the time of slaughter between March 2016 and October 2017. All samples were negative for CSF virus $\mathrm{Ab}$ on ELISA. Because of uncertainty in the sensitivity of the CSF Ab ELISA, estimates of the true prevalence of CSF were calculated using Bayesian methods. The median and upper bound of the 95\% credible intervals for the true prevalence of CSF was zero when the diagnostic sensitivity of the CSF Ab ELISA was assumed to range from 0.75 to 0.95 . These results provide evidence that the population of domestic pigs in Victoria in 2016-2017 was free of CSF.
\end{abstract}

Key Words: Pigs, classical swine fever, disease freedom, Victoria

This article is protected by copyright. All rights reserved. 


\section{Introduction}

Classical swine fever (CSF), also known as hog cholera, is a highly contagious disease of pigs $^{1,2}$ caused by a pestivirus. In its acute form, typical clinical signs can include fever, hyperaemia or cyanosis of the extremities, loss of appetite, vomiting, abortions, convulsions and/or a stiff gait and eventual death. ${ }^{3}$ In the chronic form, clinical signs are generally milder and persist for 3 to 4 weeks. The incubation period of pigs infected postnatally is 2 to 14 days (up to 3 months in chronic cases) while pigs exposed prenatally may be persistently infected throughout life. In infected holdings, up to $43 \%$ of pregnant sows may become carriers. ${ }^{4}$ Infection can result in high within-holding mortality rates and substantial economic losses due to reductions in productivity and increased feed costs. ${ }^{5}$

The most likely way that CSF virus might enter Australia is by illegally imported infected pig meat/tissue being fed deliberately or inadvertently to commercial or feral pigs ('swill' feeding) or from illegally imported genetic material. ${ }^{4}$ CSF also has the potential to become established in Australian feral pig populations if it were introduced into northern Australia from the Indian subcontinent or from West Papua where the disease is endemic. ${ }^{6,7}$

The Australian pig herd is comprised of approximately 237,000 sows, producing over 4.7 million pigs for slaughter annually. ${ }^{8}$ Approximately $90 \%$ of Australia's pork production comes from intensive, commercial piggeries but the industry also includes substantial numbers of free-range and smallholder piggeries. CSF establishment in Australia could result initially in significant within-holding mortalities leading to a rapid structural change within the local pig industry, with concomitant social and economic dislocation. ${ }^{4}$ 
Classical swine fever is listed as a notifiable disease by the OIE (the World Organisation for Animal Health) ${ }^{9}$ and in Australia by all jurisdictions. ${ }^{10}$ In Australia a notifiable disease is a disease that must be reported within a defined timeframe to relevant veterinary authorities if the presence of clinical signs are known or suspected in an animal or group of animals. CSF outbreaks occurred in Australia in 1903, 1927-1928, 1942-1943 and 1960-1961, and on each occasion the disease was quickly eradicated; Australia is currently considered free of CSF. OIE member countries that are free of CSF are expected to confirm their CSF freedom status annually. ${ }^{11}$ Current CSF surveillance in the pig industry in Victoria relies on the legal obligation of holding managers and/or their veterinarians to notify authorities of suspicion of the disease, as well as an industry-funded on-farm disease investigation program. ${ }^{12,13}$ To augment this largely 'passive' surveillance portfolio, a one-off, proactive, targeted surveillance project to assess the level of exposure of domestic pigs to CSF virus was developed.

\section{Materials and methods}

Developing a clear definition for a pig "herd" within Victoria, applicable to smallholders or small commercial or large commercial herds, proved problematic, with potential applications of a Property Identification Code (PIC), ear tattoo and/or owner details, as one or more herd identifiers. Therefore, for the purpose of this paper, all pigs sourced from the same PIC were defined using the term "holding”, with acknowledgement that multiple management groups could be listed under the same holding/PIC.

To determine the appropriate number of pigs to test to be able to declare the Victorian pig industry free from CSF at a defined level of confidence, holding and individual animal design 
prevalence estimates were developed as follows. We assumed that if a moderate to highly virulent strain of CSF virus was present in Victorian domestic pigs, within-holding mortality rates would be greater than $25 \%$ (i.e. approximately 20-50 days after introduction of CSF virus) ${ }^{14}$ while the prevalence of infected holdings would initially be low (less than $10 \%$ ). We acknowledge that, if a low virulent strain of CSF virus was present, such as that which occurred in New South Wales in 1960-1961, within-holding mortality rates may be substantially lower than $25 \% .{ }^{15}$ The sample size for a two-stage serological survey to demonstrate freedom from CSF virus at a confidence of 95\% and holding-level sensitivity of 90\%, assuming a conservative animal-level test (Ab ELISA) sensitivity of $80 \%$, was calculated as 32 holdings with 12 animals to be sampled per holding (i.e. the number of samples required to meet the objectives of the survey was $32 \times 12=384){ }^{a}$

Pig movements to abattoirs between March 2016 and October 2017 provided estimates of the number and the size of pig holdings operating in Victoria. These data were cross-referenced with the latest available industry data (2012-2013) sourced from Australian Pork Limited. ${ }^{8}$ Industry records showed that there were 396 pig herds operating in Victoria in 2012-2013, whereas 432 individual herds moved pigs to abattoirs for the period 2016-2017. An assumption was made that, for the purposes of this paper, a 'herd' was approximately equivalent to a 'holding'. Therefore, this provided confidence that movement-to-abattoir records were an appropriate source of information to estimate both the number of commercial pig holdings operating in Victorian and the approximate size of each holding.

\footnotetext{
${ }^{\mathrm{a}}$ http://epitools.ausvet.com.au/content.php?page=2StageFreedomSS 2
} 
Three hundred and seventy-five holdings sold pigs to an abattoir during the period March 2016 to October 2017. Each holding was categorised into one of three classes based on the number of slaughtered pigs throughout the study period (small: $\leq 50$, medium: 51 to 500 and large: $>500$ adult pigs). It was assumed that these categories represented each of the domestic pig farming operations present in Victoria, namely smallholders, small commercial piggeries and large commercial piggeries. The proportion of holdings to be sampled from each holding category (i.e. small, medium and large) was then estimated based on pig movement-toabattoir figures to determine the number of holdings from each category to be sampled. Holdings were further categorised by their geographic location, based on the three regions used by Agriculture Victoria, namely the Northern, South-West and South-East regions (Figure 1; Table 1).

A single blood sample was collected from each pig at the point of slaughter at all commercial abattoirs servicing piggeries across Victoria between March 2016 and October 2017. Abattoirs were visited approximately every two weeks as part of a routine herd health program, with holdings targeted opportunistically by Agriculture Victoria staff based on known holding size. The holding identifier derived from each slaughtered pig's identification tattoo brand or ear tag was recorded at the time of blood sample collection. The geographic location of the holding (to the shire-level) was verified by cross-checking each holding identifier with details held by the Agriculture Victoria Animal Disease Management Information System (ADMIS). As samples were taken opportunistically at the time of slaughter, holding size was unknown at the time of sampling. Holding size was estimated 
post collection by referencing ADMIS or the slaughter records for each holding that were accumulated during the study period.

Testing for exposure to CSF virus was carried out using the available commercial Ab ELISA (manufacturer: IDEXX) at the Centre for AgriBioscience (Victorian State Veterinary Laboratory; Bundoora; Victoria; Australia). The CSF Ab ELISA has a diagnostic specificity of 1.00 (95\% CI [confidence interval] 0.997, 1.00) but an unconfirmed diagnostic sensitivity. ${ }^{16}$

The true prevalence of CSF Ab ELISA positivity, taking into account uncertainty in the diagnostic sensitivity of the CSF Ab ELISA was determined using the approach described by Rogan and Gladen (1978) and modified for the low prevalence situation using Bayesian methods, as described by Messam et al. (2008). Using a Bayesian approach, prior distributions for each of the unknown parameters, including the estimated CSF prevalence were specified and reflected known information. Our prior estimate of the holding-level prevalence of CSF Ab ELISA positivity was set to 0.10, with 95\% certainty that the holdinglevel prevalence was greater than 0.01 . Our prior estimate of the within-holding prevalence of CSF Ab ELISA positivity in holdings that were infected was set to 0.25 , with $95 \%$ certainty that the within-holding prevalence was greater than 0.10 .

Analyses were carried out assuming the most likely value of the diagnostic sensitivity of the CSF Ab ELISA was 0.75, 0.80, 0.85, 0.90 and 0.95. For each analysis, priors were set to reflect the assumption that we were $80 \%$ certain that the diagnostic sensitivity was greater than 0.15 less than the most likely value.

This article is protected by copyright. All rights reserved. 
Bayesian analyses were run using JAGS via the contributed R package runjags. ${ }^{19-21}$ We ran the Markov chain Monte Carlo (MCMC) sampler for 100,000 iterations and discarded the first 5,000 ‘burn-in’ samples. Two parallel chains were run using diverse initial values to ensure that convergence was achieved to the same distribution. Convergence was visually assessed by plotting cumulative path plots for each of the monitored parameters (true prevalence and diagnostic sensitivity). Results are reported as the median true prevalence of CSF Ab ELISA seropositivity and their 95\% credible intervals.

\section{Results}

Three hundred and ninety-one pigs were sampled from 23 holdings (Table 2). Samples were predominately collected during the months of spring (September, October, November; $\mathrm{n}=$ 13) and autumn (March, April, May; $n=5)$, with the remaining herds sampled in August $(n=$ 5). All abattoirs that slaughter pigs in Victoria were visited during the study. The Northern region of Victoria and large holdings of $>500$ pigs were over-represented (Figure 1). All of the 391 samples were negative to the CSF Ab ELISA test, resulting in an apparent prevalence of CSF virus in Victorian domestic pigs of $0.0 \%$ (95 CI: 0.0\% to 9.4\%).

Figure 2 is a box and whisker plot showing the distribution of CSF Ab ELISA prevalence estimates for the 200,000 iterations of the MCMC sampler run for each estimate of CSF Ab ELISA diagnostic sensitivity. The point estimate and upper bound of the $95 \%$ credible intervals for the true prevalence of CSF was zero when the diagnostic sensitivity of the CSF Ab ELISA was assumed to range from 0.75 to 0.95 (Figure 2). In nine of the $5 \times 200,000=$ 1,000,000 MCMC iterations the estimated CSF Ab ELISA prevalence was greater than zero. 
When the sensitivity of the CSF Ab ELISA was 0.95, none of the 200,000 iterations of the MCMC sampler returned a prevalence greater than zero.

\section{Discussion}

The point estimate and the upper bound of the $95 \%$ credible interval for the true prevalence of CSF were zero assuming the sensitivity of the CSF Ab ELISA ranged from 0.75 to 0.95, further strengthening a claim that the population of domestic pigs in Victoria in 2016-2017 was free of CSF. As shown in Figure 2, a CSF prevalence of greater than zero was returned in only nine of the 1,000,000 MCMC iterations. Of note is that none of the iterations returned a prevalence of greater than zero when the sensitivity of the CSF Ab ELISA was assumed to be 0.95 . This is because when diagnostic test sensitivity is high, there will be small numbers of disease positive-test negative pigs, increasing the certainty that disease is absent when all tests return a negative result.

The estimated lower credible interval limit of the diagnostic specificity of the CSF Ab ELISA was 0.997 which meant that false-positive results were unlikely (but possible) when testing 300-400 truly non-infected animals. Clear protocols for resolving animal and holding status in the event of finding one or more test-positive animals need to be agreed with stakeholders before a survey like this is undertaken. In this study, follow-up procedures were planned but were not activated because none of the animals that were tested returned a positive test result. It is acknowledged that, even though the study population exceeded the calculated sample size, the number of holdings sampled was less than that required. Although the study results

presented provide evidence towards disease freedom, it is possible that the number of 
holdings sampled represented an incomplete coverage of the population and, so, the desired population sensitivity (confidence) of 95\% was over-estimated.

Although sampling at the point of slaughter is relatively easy and low cost, this method is inherently biased as only animals that are healthy enough for slaughter will be sampled. Animals that may carry CSF virus without visible clinical signs, such as breeding sows, will not be sampled using this methodology, leading to a potentially biased disease-free status. However, as CSF typically manifests with high morbidity and mortality, it is unlikely that carrier animals would be present in a naïve population, such as the Australian pig herd, without infection also being diagnosed in slaughtered pigs. This situation is more likely after eradication methods had been employed and surveillance within the remaining pig population is required to prove disease freedom. Only Victorian pigs slaughtered in Victorian abattoirs were sampled. Victorian pigs sent to interstate abattoirs were not sampled. The current disease on-farm investigation program will continue to underpin Victoria's claim to CSF freedom.

An alternative to structured representative sero-prevalence surveys, such as that described in this study, is the use of scenario trees to combine data from multiple sources collected over extended periods of time to support claims of disease freedom. ${ }^{22-24}$ This methodology can provide a comprehensive coverage of the target population and allow calculation of the overall surveillance system sensitivity, including both passively collected surveillance data and data from targeted surveys. In the case of CSF, the current on-farm passive disease investigation program could be combined with on-going abattoir sampling. ${ }^{25}$ This approach would decrease the need for costly structured surveys and would allow Agriculture Victoria 
to determine if multiple surveillance activities are sufficient to achieve the target confidence in disease freedom.

This study did not include testing of feral pigs for CSF virus and it is noted that sampling of this population would provide further confidence in Victoria's CSF disease freedom status. Actively training Victorian pig hunters to collect samples and provide animal demographic and details of the location of feral pigs at the time of slaughter would assist this process.

\section{Acknowledgements}

The authors acknowledge funding provided by Agriculture Victoria; Department of Economic Development, Jobs, Transport and Resources (DEDJTR); Victoria; Australia. The authors wish to thank Mr. Ian Shurvell (Agriculture Victoria) who developed Figure 1 and abattoir management for access to sample material.

\section{Conflict of interest and funding}

The authors had no conflicts of interest to declare. Funding was provided for the Department of Economic Development, Jobs, Transport and Resources.

\section{References}

1. Schembri N, Hernandez-Jover M, Toribio J-A, Holyoake P. On-farm characteristics and biosecurity protocols for small-scale swine producers in eastern Australia. Preventive Veterinary Medicine 2015;118:104-116.

2. Brookes V, Hernandez-Jover M, Cowled B, Holyoake P, Ward MP. Building a picture: Prioritization of exotic diseases for the pig industry in Australia using multi-criterion decision analysis. Preventive Veterinary Medicine 2014;113:103-117.

3. Postel A, Austernmann-Busch S, Petrov A, Moennig V. Epidemiology, diagnosis and control of classical swine fever: Recent developments and future challenges. Transboundary and Emerging Diseases 2017:doi: 10.1111/tbed.12676.

This article is protected by copyright. All rights reserved. 
4. Anonymous. AUSVETPLAN: Disease strategy - Classical Swine Fever Version 4.0. In: National Biosecurity Committee, editor. Australian Veterinary Emergency Plan, 2015.

5. Garner MG, Whan I, Gard G, Philips D. The expected economic impact of selected exotic diseases on the pig industry of Australia. Revue Scientifique et Techique Office International des Epizooties 2001;20:671-685.

6. Cowled B, Garner M, Negus K, Ward MP. Controlling disease outbreaks in wildlife using limited culling: modelling classical swine fever incursions in wild pigs in Australia. Veterinary Research 2012;43:doi: 10.1186/1297-9716-1143-1183.

7. Leslie E, Cowled B, Garner M, Toribio J-A, Ward MP. Effective surveillance strategies following a potential Classical Swine Fever incursion in a remote wild pig population in north-western Australia. Transboundary and Emerging Diseases 2014;61:432-442.

8. Australian Pork Limited. Section 1: Structure of the Australian Pork Industry. In: http://auspigannual.realviewdigital.com/\#folio=OFC, editor. Australian Pig Annual 2012-13, 2013.

9. World Organization for Animal Health (OIE). Chapter 2.8.3. Classical Swine Fever (hog cholera). Manual of Diagnostic Tests and Vaccines for Terrestrial Animals. OIE, Paris, France, 2014.

10. Agriculture Victoria. Notifiable Diseases. In: Victoria A, editor, http://agriculture.vic.gov.au/agriculture/pests-diseases-and-weeds/animaldiseases/notifiable-diseases, 2018.

11. World Organization for Animal Health (OIE). Classical Swine Fever. 2018. Retrieved 9 April 2018.

12. State Government of Victoria. Livestock Disease Control Act 1994. Version No. 058 edn,

http://www.legislation.vic.gov.au/Domino/Web_Notes/LDMS/LTObject_Store/LTObjSt3.nsf /DDE300B846EED9C7CA257616000A3571/AFB12F519A3B9867CA2577610024A83C/\$FILE/9 4-115a058.pdf, 2010.

13. Agriculture Victoria. Significant Disease Investigation (SDI) program. In: Agriculture Victoria, editor, http://agriculture.vic.gov.au/agriculture/pests-diseases-and-weeds/animaldiseases/disease-surveillance-programs/significant-disease-investigation-sdi-program, 2018. 14. Stark K, Pfeiffer D, Morris R. Within-farm spread of classical swine fever virus - a blueprint for a stochastic simulation model. Veterinary Quarterly 2000;22:36-43.

15. Geering W, Forman A. Exotic diseases. In: Department of Primary Industries and Energy, editor. Animal Health in Australia. Bureau of Rural Science Commonwealth Scientific and Industrial Research Organization, Geelong, 1987.

16. Anonymous. IDEXX CSFV Ab Test: Accurate detection of antibodies against classical swine fever (CSFV). In: http://www.idexx.com.au/pdf/en_au/livestock-poultry/csfv-ab-testinformation-sheet.pdf, editor. IDEXX Laboratories, Westbrook, Maine, 2010.

17. Rogan W, Gladen B. Estimating prevalence from results of a screening test. American Journal of Epidemiology 1978;107:71-76. 
18. Messam L, Branscum A, Collins M, Gardner I. Frequentist and Bayesian approaches to prevalence estimation using examples from Johne's disease. Animal Health Research Reviews 2008;9:1-23.

19. Plummer M. JAGS: A program for analysis of Bayesian graphical models using Gibbs sampling. http://mcmc-jags.sourceforge.net/, 2003.

20. R Core Team. R Core Team: A language and environment for statistical computing. $\mathrm{R}$ Foundation for Statistical Computing, Vienna, Australia, 2018.

21. Denwood M. runjags: An R Package Providing Interface Utilities, Model Templates, Parallel Computing Methods and Additional Distributions for MCMC Models in JAGS. Journal of Statistical Software 2016;71:1-25.

22. Martin P, Cameron A, Greiner $M$. Demonstrating freedom from disease using multiple complex data sources 1: A new methodology based on scenario trees. Preventive Veterinary Medicine 2007;79:71-97.

23. Christensen J, Vallieres A. Scenario tree model for animal disease freedom framed in the OIE context using the example of a generic swine model for Aujeszky's disease in commercial swine in Canada. Preventive Veterinary Medicine 2016;123:60-70.

24. Blickenstorfer $\mathrm{S}$, Schwermer $\mathrm{H}$, Engels $\mathrm{M}$ et al. Using scenario tree modelling for targeted herd sampling to substantiate freedom from disease. BMC Veterinary Research 2011;7:49-58.

25. Martin P, Cameron A, Barfod K, Sergeant E, Greiner M. Demonstrating freedom from disease using multiple complex data sources 2: Case study - Classical swine fever in Denmark. Preventive Veterinary Medicine 2007;79:98-115.

This article is protected by copyright. All rights reserved. 
Table 1. Required number of pig holdings (and number of pigs) to be sampled for the classical swine fever serological survey (a total of 32 holdings and 246 pigs) stratified by Agriculture Victoria region and holding size.

\begin{tabular}{lcccc}
\hline \multirow{2}{*}{ Holding size } & \multicolumn{3}{c}{ Region } & \multirow{2}{*}{ Total } \\
\cline { 2 - 4 } & Northern & South-West & South-East & \\
\hline Small ( $\leq 50$ pigs) & $3(24)$ & $13(104)$ & $2(16)$ & $18(144)$ \\
Medium (51-500 pigs) & $2(16)$ & $2(16)$ & $1(8)$ & $5(40)$ \\
Large (>500 pigs) & $5(30)$ & $3(24)$ & $1(8)$ & $9(62)$ \\
Total & $10(70)$ & $18(144)$ & $4(32)$ & $32(246)$ \\
\hline
\end{tabular}

This article is protected by copyright. All rights reserved. 
Table 2. Actual number of pig holdings (and total number of pigs) sampled for the classical swine fever serological survey (March 2016 - October 2017) (a total of 23 holdings and 391 pigs) stratified by Agriculture Victoria region and holding size.

\begin{tabular}{lcccc}
\hline \multicolumn{1}{c}{ Holding size } & \multicolumn{3}{c}{ Geographic region } & \multirow{2}{*}{ Total } \\
\hline Small ( $\leq 50$ pigs) & $2(20)$ & $1(10)$ & - & $3(30)$ \\
Medium (51-500 pigs) & $4(98)$ & $2(24)$ & $2(8)$ & $8(130)$ \\
Large (> 500 pigs) & $7(129)$ & $4(80)$ & $1(22)$ & $12(231)$ \\
Total & $13(247)$ & $7(114)$ & $3(30)$ & $23(391)$ \\
\hline
\end{tabular}

This article is protected by copyright. All rights reserved. 
Figure 1. Geographic location of study holdings in Victoria $(n=23)$ sampled for exposure to classical swine fever virus (March 2016 - October 2017), with holding size denoted by icon size ('small': $\leq 50$ pigs; 'medium': 51-500 pigs; 'large': >500 pigs). Note: holdings of the same size within the same Shire overlap.

This article is protected by copyright. All rights reserved. 
Figure 2. Box and whisker plot showing the distribution of the estimated true prevalence of CSF positive pigs (from the 200,000 MCMC iterations) as a function of estimates of the diagnostic sensitivity of the CSF Ab ELISA. Ninety-five percent credible intervals of the estimate of the true prevalence of CSF and the maximum true prevalence estimate (from 200,000 iterations of the MCMC sampler) are indicated for each estimate of Ab ELISA sensitivity. The boxes are not evident because most of the CSF true prevalence estimates were zero. 


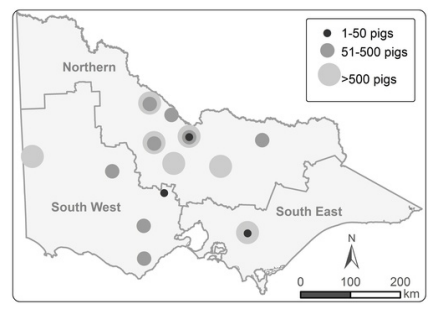

AVJ_12871_Figure1.jpg

This article is protected by copyright. All rights reserved. 


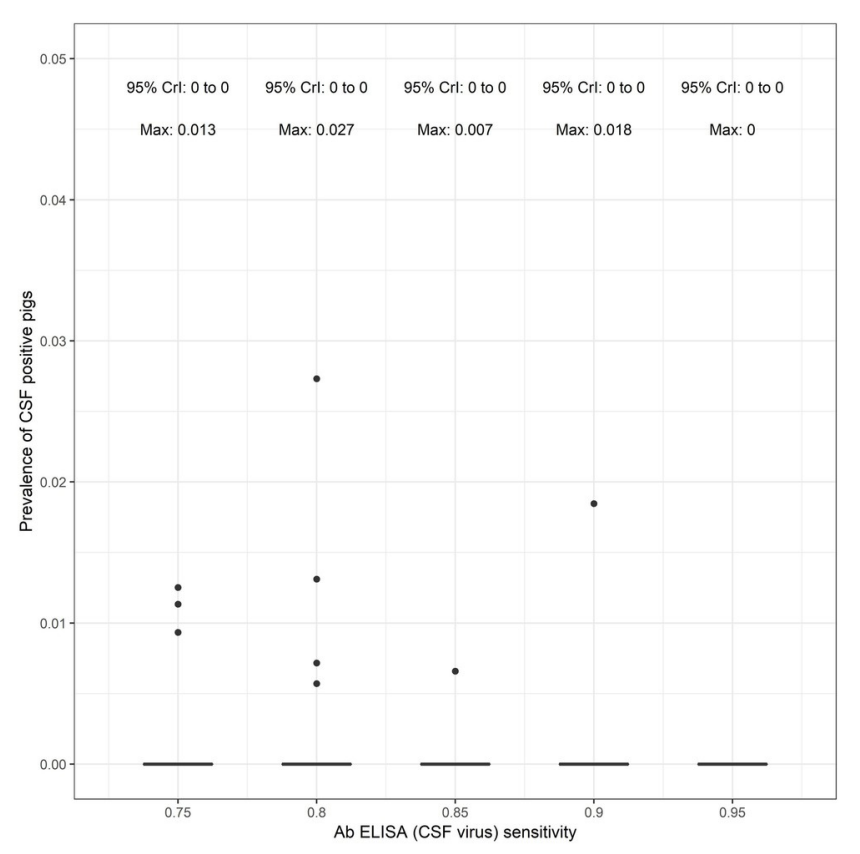

AVJ_12871_Figure2.jpg

This article is protected by copyright. All rights reserved. 


\section{University Library}

\section{- M M N E R VA A gateway to Melbourne's research publications}

Minerva Access is the Institutional Repository of The University of Melbourne

Author/s:

Hunnam, JC;Moore, KM;Daniel, P;Stevenson, MA;Salmon, SE

Title:

Classical swine fever in Victorian domestic pigs: evidence of disease freedom

Date:

2019-11-01

Citation:

Hunnam, J. C., Moore, K. M., Daniel, P., Stevenson, M. A. \& Salmon, S. E. (2019). Classical swine fever in Victorian domestic pigs: evidence of disease freedom. AUSTRALIAN VETERINARY JOURNAL, 97 (11), pp.447-451. https://doi.org/10.1111/avj.12871.

Persistent Link:

http://hdl.handle.net/11343/286358 